\title{
LIFE PREDICTION FOR LY12CZ NOTCHED PLATE BASED ON THE CONTINUUM DAMAGE MECHANICS AND THE GENETIC ALGORITHM AND RADIAL BASIS FUNCTION METHOD
}

\author{
Jiaying Gao, Peng Li \\ School of Aeronautics Science and Engineering, Beihang University, Beijing, China \\ e-mail: gaojiaying_1988@163.com; lpeng210@sina.com (corresponding author)
}

\begin{abstract}
In this paper, a new method based on the Continuum Damage Mechanics (CDM) and the Genetic Algorithm and Radial Basis Function neural network method (GARBF) is proposed to predict fatigue life of LY12CZ notched plate. Firstly, the multiaxial fatigue damage evolution equation is derived, and the fatigue life of the notched specimen is predicted based on the CDM method. Secondly, the RBF method is introduced to modify the relative deviation between the theoretical result and actual life. According to the drawbacks of the RBF method, the GA is adopted to optimize network parameters to effectively improve the model quality and reduce the training error. Then, the verification test indicates that the combined method of CDM and GARBF is able to reduce the average relative error of the results of fatigue life prediction to about $7 \%$, which shows that the new method to predict the fatigue life is more reliable. At last, compared with the predicted results of the traditional Back Propagation (BP) neural network, the GARBF model proposed in this paper has a better optimization effect and the result is more stable. This research provides a feasible way to predict the fatigue lives of the notched plate based on the CDM and GARBF method.
\end{abstract}

Keywords: life prediction, CDM, LY12CZ, notched plate, GARBF

\section{Introduction}

In practical applications, many engineering structures are subject to cyclic loads, and fatigue damage is a common form of failure (Schijve, 2001; Zhan et al., 2013). Fatigue damage is a progressive and localized material behavior which occurs when a structure component is subjected to cyclic loading. Fatigue cracks are prone to initiate at the positions of notches and geometrical irregularity due to stress concentration. The notch is considered as one of the most important problems in the design of structure components. Different from the uniaxial fatigue problem, the fatigue damage of a notched component involves multiaxial stress and strain states. So, it is important to present a method for fatigue life prediction of notched plates.

In the past decades, many researchers have been focused on the fatigue experiment and statistic analysis (Suresh, 1998), which is a general method of fatigue life prediction in engineering. However, this method takes too much time and sometimes the result is not satisfactory. So, it is necessary to find an easy and reliable method. The critical plane approach (Karolczuk and Macha, 2005) and stress invariant approaches (Li et al., 2000) have been widely used in researches and applications. However, these approaches often fail to capture the fatigue damage evolution such as material degradation due to damage. Fatigue accumulation damage theory (Lemaitre and Chaboche, 1990; Zhan et al., 2015b, 2016, 2017c) is considered to be one of the most effective methods for fatigue life prediction. The Continuum Damage Mechanics (CDM) provides an effective method to describe degradation of mechanical properties of materials, which take into account damage evolution using the concept of effective stress and has been widely used in 
recent years (Movaghghar and Lvov, 2012; Upadhyaya and Sridhara, 2012; Zhang et al., 2015a, 2017a,b).

While the methods based on the damage law are carried out, such problems also attract some researchers engaged in the machine learning field. They attempt to take the advantages of machine learning theories in complex system modeling to enhance the accuracy of fatigue life prediction. Pujol and Pinto (2011) proposed a novel method based on neural network to predict the fatigue life. Guo et al. (2014) introduced the support vector machine (SVM) method to predict the life of the packaging EMC material. Liu and Xuan (2008) adopted the rough sets theory to analyze important affecting parameters on low cycle fatigue life. The fatigue life prediction is a complicated problem. There is a large amount of non-linear and non-smooth factors that rapidly influence the actual material life. Compared to traditional theoretical methods, the artificial neural network (ANN) method can better overcome these difficulties (Gao et al., 2015, 2016; Monteiro et al., 2016; Nagarajan and Jonkman, 2013; Reid etal, 2013). Introducing the ANN method into the solution of fatigue life prediction has several advantages: (1) ANN is able to approximate any non-linear and non-smooth function with any accuracy; (2) ANN has strong anti-interference ability and good robustness; (3) The ANN structure is suitable for parallel data processing, and the computation is efficient and fast; (4) ANN has good generalization ability. In this paper, the Radial Basis Function (RBF) neural network method is adopted to predict the fatigue life for LY12CZ notched plate. Some researches have shown that the RBF neural network has better optimal approximation ability than the traditional Back Propagation neural network (BPNN). Compared to BPNN, the RBF model has fewer parameters to be learnt. The RBF neural network is composed of 3 layers: an input layer, a hidden layer and an output layer. After suitable data pre-processing, the data will be imported into the hidden layer. The hidden layer consists of several neurons, and each neuron can be regarded as a cluster center which is able to generate the local response by the inputs. When the distance between the inputs and the cluster center are close enough, the hidden neuron will make a non-zero response. The final output of the RBF network is equal to a linear combination of all hidden layer neuron responses.

There are also some defects in the use of the RBF method. The RBF is not good at global searching and is easy to fall into the local minimum. In this paper, the Genetic Algorithm (GA) is adopted to optimize the training process of the RBF model. The GA is developed based on the biological evolution process. Several biological concepts such as reproduction, crossover, mutation, competition are introduced into the GA algorithm. The GA will adjust and improve a series of feasible solutions in the process of evolution, and finally find the optimum solution in the whole multi-dimensional space. The GA is a global optimization algorithm, so it can effectively overcome the drawbacks of traditional methods (Camacho-Vallejo et al., 2015; Goldberg and Samtani, 2015; Zhan et al., 2015c).

In this paper, the continuum damage mechanics (CDM) and an optimization model composed of the Genetic Algorithm and Radial Basis Function neural network method (GARBF) are proposed to conduct the fatigue life prediction for LY12CZ notched plate in the case of cyclic loading using the framework is shown in Fig. 1. Firstly, the multiaxial fatigue damage evolution equation is derived. Secondly, according to the fatigue experimental data, the material parameters in the damage evolution equation are identified. Then the fatigue life prediction of the notched specimen is conducted. On the basis of the CDM method, the Radial Basis Function (RBF) method is introduced to modify the relative deviation between the theoretical result and actual life. In addition, the Genetic Algorithm (GA) is adopted to improve the RBF training effect in order to obtain a more reliable optimization model (GARBF). Finally, the verification test indicates that the combined method of CDM and GARBF is able to reduce the average relative error of fatigue life prediction to about $7 \%$, and the life prediction result is more reliable. Compared to the traditional backpropagation (BP) neural network, the GARBF model proposed in this paper has a better optimization effect and the result is more stable. 


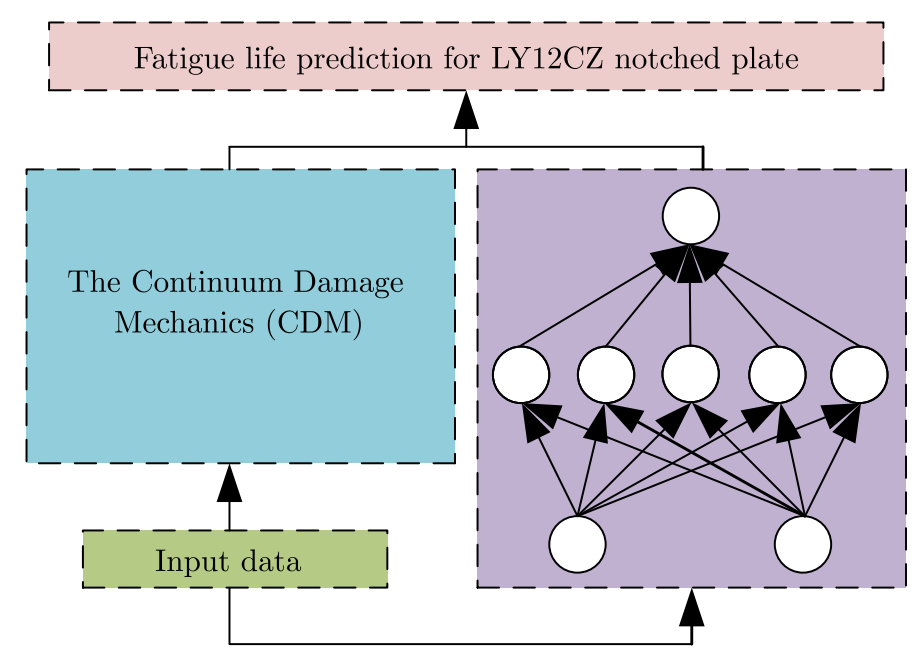

Fig. 1. The framework of the methodology

\section{The fatigue damage evolution model}

In uniaxial cycle loading, based on the remaining life and continuum damage concepts, the fatigue cumulative damage model can be illustrated as (Zhan et al., 2015c)

$$
\dot{D}=\frac{d D}{d N}=\left[1-(1-D)^{\beta+1}\right]^{\alpha\left(\sigma_{\max }, \sigma_{m}\right)}\left[\frac{\sigma_{\max }-\sigma_{m}}{M\left(\sigma_{m}\right)(1-D)}\right]^{\beta}
$$

where $D$ is the damage scalar variable and $N$ is the number of cycles. $\sigma_{\max }$ and $\sigma_{m}$ are, respectively, the maximum and mean applied stress. $\beta$ is a material parameter. The expression of $\alpha\left(\sigma_{\max }, \sigma_{m}\right)$ is defined as

$$
\alpha\left(\sigma_{\max }, \sigma_{m}\right)=1-a\left\langle\frac{\sigma_{\max }-\sigma_{f}\left(\sigma_{m}\right)}{\sigma_{u}-\sigma_{\max }}\right\rangle
$$

where $\sigma_{u}$ is the ultimate tensile stress, $\sigma_{l 0}$ is the fatigue limit for fully reversed conditions. $a$ and $b_{1}$ are material parameters. The expression of $M\left(\sigma_{m}\right)$ is defined as

$$
M\left(\sigma_{m}\right)=M_{0}\left(1-b_{2} \sigma_{m}\right)
$$

where $M_{0}$ and $b_{2}$ are material parameters. The number of cycles to failure $N_{F}$ for a constant stress condition is obtained by integrating Eq. (2.1) from $D=0$ to $D=1$, leading to

$$
N_{F}=\frac{1}{1+\beta} \frac{1}{a M_{0}^{-\beta}} \frac{\left\langle\sigma_{u}-\sigma_{\max }\right\rangle}{\left\langle\sigma_{\max }-\sigma_{f}\left(\sigma_{m}\right)\right\rangle}\left(\frac{\sigma_{a}}{1-b_{2} \sigma_{m}}\right)^{-\beta}
$$

where $\sigma_{a}$ is the stress amplitude during one loading cycle.

In the practical engineering application, the stress and strain are always multiaxial. The damage evolution law in the case of multiaxial loading is given as follows

$$
\dot{D}=\frac{d D}{d N}=\left[1-(1-D)^{\beta+1}\right]^{\alpha}\left(\frac{A_{I I}}{M_{0}\left(1-3 b_{2} \sigma_{H, m}\right)(1-D)}\right)^{\beta}
$$

where $A_{I I}$ is the amplitude of octahedral shear stress and $\sigma_{H, m}$ is the mean hydrostatic stress. The parameter $\alpha$ is defined by

$$
\alpha=1-a\left\langle\frac{A_{I I}-A_{I I}^{*}}{\sigma_{u}-\sigma_{e, \max }}\right\rangle
$$


where $\sigma_{e, \max }$ is the maximum equivalent stress which is calculated by maximising the von Mises stress over a loading cycle. The Sines fatigue limit criterion $A_{I I}^{*}$ in this model is formulated by

$$
A_{I I}^{*}=\sigma_{l 0}\left(1-3 b_{1} \sigma_{H, m}\right)
$$

By integrating of Eq. (2.5) from $D=0$ to $D=1$ for a constant stress condition, the number of cycles to failure $N_{F}$ is

$$
N_{F}=\frac{1}{1+\beta} \frac{1}{a M_{0}^{-\beta}} \frac{\left\langle\sigma_{u}-\sigma_{e, \max }\right\rangle}{\left\langle A_{I I}-A_{I I}^{*}\right\rangle}\left(\frac{A_{I I}}{1-b_{2} \sigma_{H, m}}\right)^{-\beta}
$$

\section{The material parameters identification}

The static properties of LY12CZ aluminium alloy and fatigue experiment results can be consulted from a handbook (Wu, 1996), and the static mechanics properties are presented in Table 1. In the modified damage evolution law, there are five parameters in the damage evolution equation. The four material parameters $\left(\beta, M_{0}, b_{1}, b_{2}\right)$ can be determined by fatigue experimental data of smooth specimens. For smooth specimens under conditions of uniaxial fatigue loading, an S-N curve has been derived. When fatigue tests are carried out at a fixed stress ratio, the relation between the number of cycles to failure $N_{F}$ and the maximum stress $\sigma_{\max }$ can be obtained. Parameters $\beta$ and $1 /\left[(1+\beta) a M_{0}^{-\beta}\right]$ come from stress-controlled $(R=-1)$ fatigue tests stress-life data. With the least square method, parameters $b_{1}$ and $b_{2}$ can be obtained from the fatigue tests data at other different stress ratios. Then the independent parameters $\beta$ and $a M_{0}^{-\beta}$ will be used in the incremental damage formulation (Zhan et al., 2017d), and $a$ is identified numerically by using the fully reversed fatigue test data for the notched specimens. Finally, the identified damage evolution parameters are listed in Table 2.

Table 1. Static properties of LY12CZ aluminium alloy

\begin{tabular}{|c|c|c|c|}
\hline$E[\mathrm{GPa}]$ & $\nu[-]$ & $\sigma_{b}[\mathrm{MPa}]$ & $\sigma_{s}[\mathrm{MPa}]$ \\
\hline \hline 73 & 0.3 & 466 & 343 \\
\hline
\end{tabular}

Table 2. Material parameters in the fatigue damage evolution equation

\begin{tabular}{|c|c|c|c|c|}
\hline$a$ & $\beta$ & $M_{0}$ & $b_{1}$ & $b_{2}$ \\
\hline \hline 0.62 & 1.76 & 712398 & 0.0001 & 0.0002 \\
\hline
\end{tabular}

\section{Fatigue life prediction for $\mathrm{LY} 12 \mathrm{CZ}$ notched plate}

Two kinds of the LY12CZ notched specimen are used and geometric profiles of which are shown in Fig. 2. The corresponding stress concentration factors $K_{T}$ are 2 and 4 , respectively. The fatigue load, stress ratio and the predicted fatigue lives under different stress levels are shown in Table 3.

\section{Introduction of an RBF neural network model}

The establishment of a theoretical model (CDM) provides an important basis and foundation for the solution of notched specimen fatigue life prediction. As shown in Table 3, there is about 20\% 
(a)

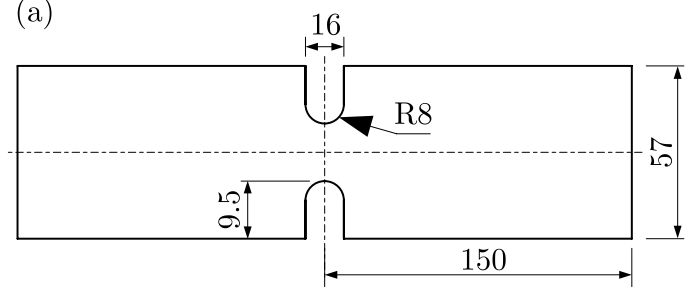

(b)

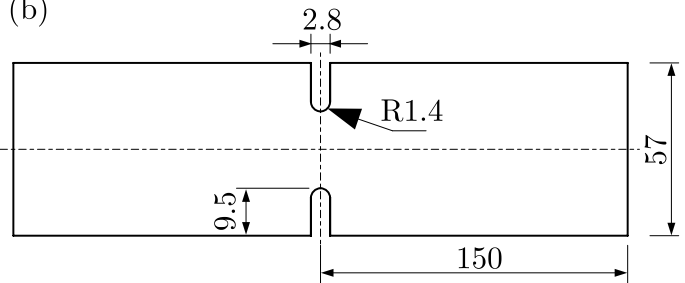

Fig. 2. The geometric profile of the notched specimen (dimensions in mm): (a) $K_{T}=2$, (b) $K_{T}=4$

Table 3. Comparisons between experimental lives and predicted results

\begin{tabular}{|c|c|c|c|c|c|}
\hline & $\begin{array}{l}\text { Stress } \\
\text { ratio }\end{array}$ & $\begin{array}{c}\sigma_{\max } \\
{[\mathrm{MPa}]}\end{array}$ & $\begin{array}{c}\text { Experimental } \\
\text { life }\end{array}$ & $\begin{array}{c}\text { Numerical } \\
\text { results }\end{array}$ & $\begin{array}{c}\text { Error } \\
{[\%]}\end{array}$ \\
\hline \multirow{10}{*}{$K_{T}=2$} & -0.2 & 175 & 34160 & 26370 & 22.80 \\
\hline & -0.09 & 155 & 114000 & 90450 & 20.66 \\
\hline & 0.02 & 137 & 217200 & 238000 & 9.58 \\
\hline & 0.25 & 112 & 1094000 & 1354000 & 23.77 \\
\hline & 0.4 & 100 & 9490000 & 8035000 & 15.33 \\
\hline & 0.4 & 300 & 85390 & 74300 & 12.98 \\
\hline & 0.60 & 262 & 226100 & 288900 & 27.77 \\
\hline & 0.68 & 250 & 778600 & 881350 & 13.20 \\
\hline & 0.75 & 240 & 2037000 & 1672000 & 17.92 \\
\hline & 0.79 & 235 & 2445000 & 1906000 & 22.04 \\
\hline \multirow{10}{*}{$K_{T}=4$} & 0.02 & 137 & 45350 & 35700 & 21.28 \\
\hline & 0.25 & 112 & 180900 & 157500 & 12.93 \\
\hline & 0.4 & 100 & 646000 & 803200 & 24.33 \\
\hline & 0.55 & 90 & 2458000 & 2079000 & 15.42 \\
\hline & 0.60 & 87 & 3319000 & 2885000 & 13.08 \\
\hline & 0.60 & 262 & 45320 & 36500 & 19.46 \\
\hline & 0.68 & 250 & 64200 & 78300 & 21.96 \\
\hline & 0.75 & 240 & 190400 & 174000 & 8.61 \\
\hline & 0.83 & 230 & 753200 & 845400 & 12.24 \\
\hline & 0.91 & 220 & 1483000 & 1727000 & 16.45 \\
\hline
\end{tabular}

deviation between the actual fatigue life and the results calculated by CDM. The deviation is mainly due to several factors: (1) some parameters in the theoretical model are not accurate; (2) there are structural defects in the theoretical model; (3) experimental environment and hardware conditions lead to certain system errors. In a word, the deviation is appeared by complicated reasons. It is difficult to develop a theoretical approach to calculate. Therefore, the RBF neural network method is adopted to modify the CDM model to obtain a more reliable fatigue life prediction result.

The RBF neural network model is composed of 3 layers: an input layer, a hidden layer and an output layer. Its topological structure is shown in Fig. 3. There are two neurons in the input layer, one stands for the stress ratio $R$ and another represents the ultimate stress $\sigma_{\max }$. In order to enhance the training efficiency and speed up the convergence rate, the input data will be standardized $\left(\bar{\sigma}_{\max }, \bar{R}\right)$ before imported into the hidden layer. Each weight from the input layer to the hidden layer is equal to 1 . The neuron number in the hidden layer is set as $h$, and each neuron represents a cluster center which is corresponding to the basis vector. The Gauss function is selected as the radial basis function. The input sample in the training set is denoted as: $\mathbf{X}=\left[x_{1}, x_{2}, \ldots, x_{i}, \ldots, x_{p}\right]^{\mathrm{T}}$, and each sample contains 2 dimension data: $x_{i}=\left(\bar{\sigma}_{\max }, \bar{R}\right)$. The 


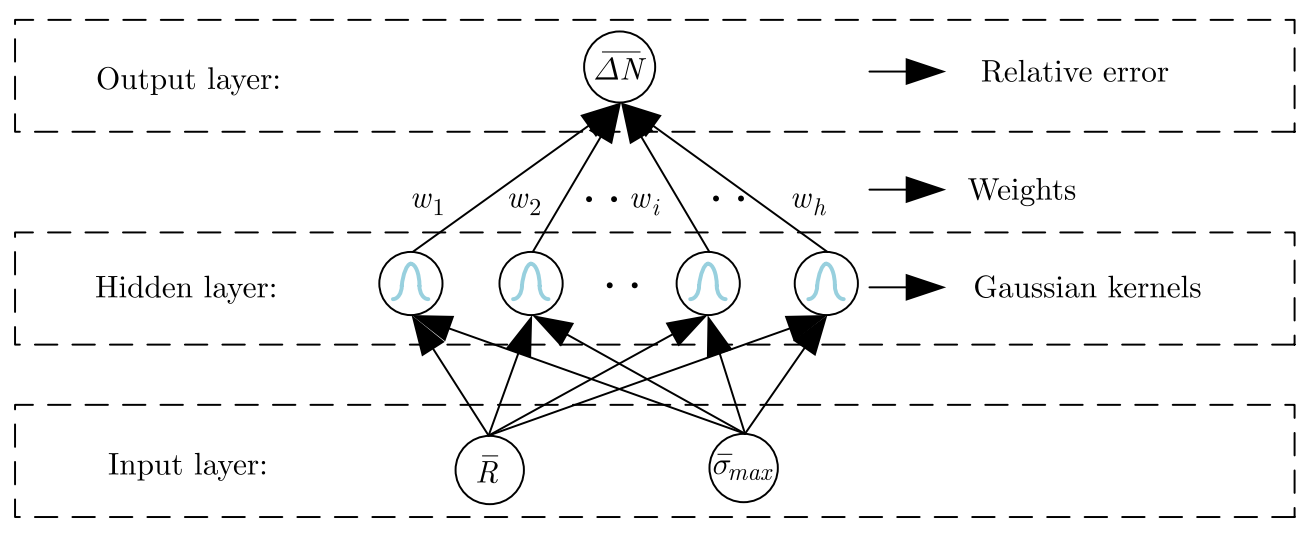

Fig. 3. The topological structure for an RBR neural network model

output label $\overline{\boldsymbol{\Delta N}}=\left[\overline{\Delta N}_{1}, \overline{\Delta N}_{2}, \ldots, \overline{\Delta N}_{i}, \ldots, \overline{\Delta N}_{p}\right]^{\mathrm{T}}$ is the relative deviation percent between the actual life $N_{A}$ and the theoretical result $N_{F}$, expressed as: $\overline{\Delta N}_{i}=\left(N_{A}-N_{F}\right) / N_{F} \cdot p$ is the total sample number in the train set. The response output $O_{1 i j}$ from the $i$-th sample to the $j$-th neuron in the hidden layer can be calculated by Eq. (5.1), in which $\sigma$ is the variance of the Gauss function, $c_{j}$ stands for the $j$-th cluster center, $\left\|\mathbf{x}_{i}-\mathbf{c}_{j}\right\|$ represents the Euclidean distance between the input vector $\mathbf{x}_{i}$ and $\mathbf{c}_{j}$

$$
O_{1 i j}=\exp \left(-\frac{1}{2 \sigma^{2}}\left\|\mathbf{x}_{i}-\mathbf{c}_{j}\right\|^{2}\right)
$$

The final output $\bar{N}$ of the RBF model is the relative deviation percent between the actual life and the theoretical result. The output is calculated by a linear combination of all Gaussian function outputs in the hidden layer. $w_{j}$ is the weight from the $j$-th neuron in the hidden layer to the output. Then, the final output for the $i$-th sample can be calculated as

$$
O_{2 i}=\sum_{j=1}^{h} w_{j} O_{1 i j}=\sum_{j=1}^{h} w_{j} \exp \left(-\frac{1}{2 \sigma^{2}}\left\|\mathbf{x}_{i}-\mathbf{c}_{j}\right\|^{2}\right)
$$

\section{The learning process for the RBF neural network model}

The learning process for the RBF model is divided into the unsupervised learning stage and the supervised learning stage.

\subsection{Unsupervised learning stage}

In the unsupervised learning stage, the $K$-means method is used to automatically cluster the sample data and locate the center of each Gaussian kernel function. The iteration step is shown as below.

Step 1. Initialization: $h$ points are random selected as the initial cluster center

$$
c_{1}\left(\bar{\sigma}_{1}, \bar{R}_{1}\right), c_{2}\left(\bar{\sigma}_{2}, \bar{R}_{2}\right), \ldots, c_{i}\left(\bar{\sigma}_{i}, \bar{R}_{i}\right), \ldots, c_{h}\left(\bar{\sigma}_{h}, \bar{R}_{h}\right)
$$

Step 2. Clustering: Each point is sorted out into the corresponding cluster by the principle of proximity. 
Step 3. Adjustment of the clustering center: The new position of each clustering center is updated by calculating the average value of sample points. If all the clustering centers are not changed any more, then the current centers will be regarded as the Gaussian kernel function centers in the hidden layer of the RBF model, the iteration ends; Else, back to Step 2.

\subsection{Supervised learning stage}

On the basis of the $K$-means clustering result in the unsupervised learning stage, the variance of the Gaussian kernel function in the hidden layer can be obtained by Eq. (6.1), in which $d_{\max }$ represents the maximum distance between the clustering centers

$$
\sigma=\frac{d_{\max }}{\sqrt{2 h}}
$$

After the determination of the clustering centers $c=\left(c_{1}, c_{2}, c_{3}, \ldots, c_{i}, \ldots, c_{h}\right)$ and the variance $\sigma$, the weight matrix can be calculated by solving the linear system in Eq. (6.2) with the least squares method

$$
\left[\begin{array}{cccc}
w_{11} & w_{12} & \cdots & w_{1 h} \\
w_{21} & w_{12} & \cdots & w_{2 h} \\
w_{31} & w_{12} & \cdots & w_{3 h}
\end{array}\right]\left[\begin{array}{c}
O_{11} \\
O_{12} \\
\vdots \\
O_{1 h}
\end{array}\right]=\left[\begin{array}{c}
y_{1} \\
y_{2} \\
y_{3}
\end{array}\right]
$$

\section{Optimization of the RBF model based on a GA algorithm}

The selection of the Gaussian kernel center is the core element of the RBF neural network design. In order to improve the neural network quality and reduce the training error, a Genetic Algorithm is adopted to optimize the initial position of each Gaussian kernel center. Chromosome encoding, fitness function selection and definition of the genetic operator are 3 parts of the GA algorithm design.

\subsection{Chromosome encoding}

When a GA is used to solve a numerical optimization problem, the binary code cannot achieve very good results. Therefore, the real number chromosome encoding is adopted, as shown in Fig. 4. Each chromosome is composed of several gene segments, and each segment represents a certain Gaussian kernel center position $c_{i}\left(\bar{\sigma}_{i}, \bar{R}_{i}\right)$.

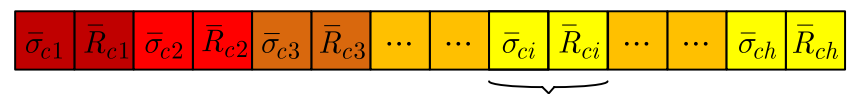

The gene segment corresponding to the $i$-th Gaussian kernel

Fig. 4. A sample of chromosome encoding

\subsection{The fitness function and the roulette selection probability}

The fitness function is used to evaluate the accuracy of calculated results. According to the previous discussion, the objective function of RBF training is expressed as Eq. $(7.1)_{1}$, and the definition of the fitness function is shown in Eq. (7.1) $)_{2}$ 


$$
E=\frac{1}{2} \sum_{i=1}^{i=p}\left(O_{2 i}-y_{i}\right)^{2} \quad \text { Fitness }=\frac{1}{E} \quad P_{k}=\frac{\text { Fitness }_{k}}{\sum_{i=1}^{i=\text { Pop }_{\text {Fitness }}} \text {. }}
$$

For the $k$-th individuals in the population, its probability of being chosen for evolution is calculated by the individual fitness and the total fitness, as shown in Eq. (7.1) ${ }_{3}$. The selection process is carried out by the roulette mode, as shown in Fig. 5. The whole disc area represents the total fitness of the population, and the fitness of each individual is corresponding to a certain sector area. The area the pointer finally stays determines which individual is selected. In this way, a higher individual fitness means a greater possibility of being chosen, while a lower fitness also has a little possibility to evolve.

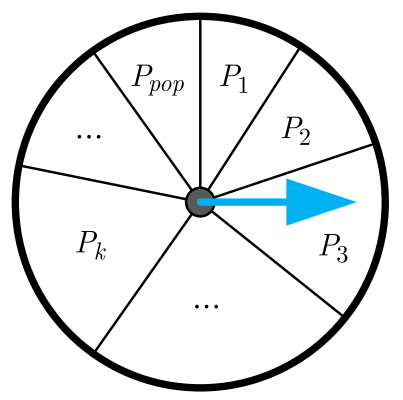

Fig. 5. The roulette choosing mode

\subsection{Genetic operator: crossover and mutation}

The purpose of the genetic operator is to preserve good chromosomes into the next generation. Assuming the crossover operator is happening on the $k$-th gene segment of the parent chromosome $c h^{i}=\left\{c h_{1}^{i}, c h_{2}^{i}, \ldots, c h_{k}^{i}, \ldots, c h_{m}^{i}\right\}$ and $c h^{j}=\left\{c h_{1}^{j}, c h_{2}^{j}, \ldots, c h_{k}^{j}, \ldots, c h_{m}^{j}\right\}$, then the real numbers of the $k$-th gene segment in the two new chromosome $\left(c h^{i s}, c h^{j s}\right)$ are calculated by Eq. (7.2), where $\psi$ is the crossover possibility and set as $80 \%$.

$$
c h_{k}^{i s}=\psi c h_{k}^{i}+(1-\psi) c h_{k}^{j} \quad c h_{k}^{j s}=\psi c h_{k}^{j}+(1-\psi) c h_{k}^{i}
$$

Each gene segment is composed of 2 normalized data: the stress ratio and the ultimate stress. Therefore, the crossover process between the parents is equivalent to calculation of two symmetric definite proportionate inserted points in a straight line defined by $\left(\bar{\sigma}_{k}^{i}, \bar{R}_{k}^{i}\right)$ and $\left(\bar{\sigma}_{k}^{j}, \bar{R}_{k}^{j}\right)$, as shown in Fig. 6.

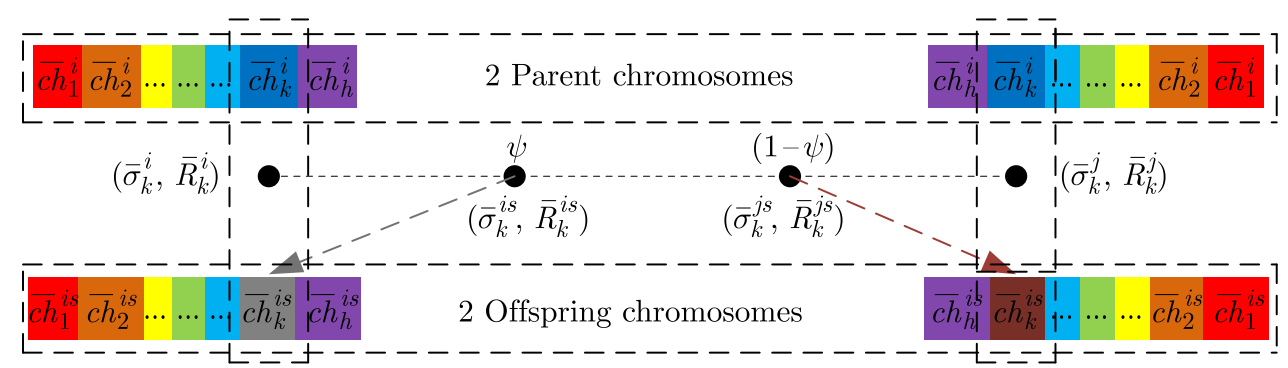

Fig. 6. The crossover process between 2 parent chromosomes

Different from binary coding, the mutation operator of real number encoding is to add a random bias $d$ to the selected gene segment, denoted as: $c h_{k}^{i s}=c h_{k}^{i}+d$. The mutation operator ensures diversity of population, and its probability is set as $3 \%$. 


\subsection{The training process of the GARBF model}

The GA algorithm is adopted to optimize the Gaussian kernel center positions in the hidden layer during the RBF training process, and the iteration step is shown as below.

Step 1. Randomly initialize the population (random selection of the clustering center position in the hidden layer), and encode the chromosomes.

Step 2. Calculate the fitness of each individual in the population, and select the optimal individual.

Step 3. If the number of generation reaches the maximum or the optimal individual satisfies the requirements of training accuracy, go to Step4; Else, the next generation of population will be produced by the 3 genetic operator: mutation, crossover and selection, then go to Step 2.

Step 4. The chromosomes are anti-encoding and the initial clustering center positions $c_{1}, c_{2}, \ldots, c_{h}$ in the hidden layer are obtained.

Step 5. The parameters of the RBF model are trained by the supervised learning method, the iteration ends.

All the experimental data are divided into two parts, $80 \%$ for training and $20 \%$ for the verification test. There is no intersection between the training set and the test set. The training error descent curve by the GARBF and RBF method are shown in Fig. 6. When the iteration epoch is less than 30, the optimization effect of the GA is not obvious, just as the red circle region shown in Fig. 7; As the iteration epoch is increasing, it is indicated that the GARBF method is superior to simple use of the RBF method in both the convergence rate and training error aspect. After about 430 epochs, the training error is reduced and converged to 0.0017.

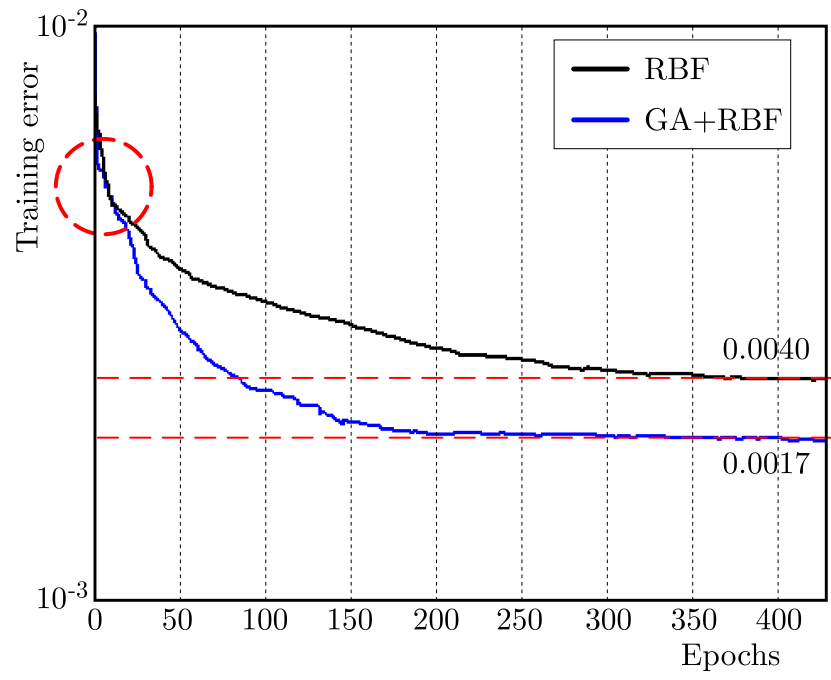

Fig. 7. The training error descent curve of GARBF and RBF models

\section{The combined method of CDM and GARBF}

The CDM and GARBF method are combined to accurately predict the fatigue life for LY12CZ notched plate. On the basis of the establishment of the CDM model, the trained GARBF model is adopted to modify the theoretical result and obtain the prediction life. In the verification 
test, $N_{F}$ represents the theoretical result calculated by the CDM model, and $N_{G A R B F}$ stands for the result after being modified by the trained GARBF model, $N_{A}$ actual life measured in the experiment. The relative error percent between $N_{F}$ and $N_{A}$ is denoted as $e_{F A}$, and the relative error percent between $N_{G A R B F}$ and $N_{A}$ is denoted as $e_{G A R B F} . e_{F A}$ and $e_{G A R B F}$ can be calculated by Eq. (8.1). Under the two conditions of $K_{T}=2$ and $K_{T}=4$, the distributions of $e_{F A}$ and $e_{G A R B F}$ are shown in Fig. 8, in which $X$ and $Y$ label respectively mean the input value of $\sigma_{\max }$ and $R$. The red circles mean the relative error exceed $20 \%$, while the blue ones mean that the relative error is less than $5 \%$. The distribution of the relative error indicates that the prediction results modified by the trained GARBF will be closer to the actual life in the whole. The combined method of CDM and GARBF proposed in this paper is effective and reliable

$$
e_{F}=\frac{N_{F}-N_{A}}{N_{F}} \cdot 100.0 \% \quad e_{G A R B F}=\frac{N_{G A R B F}-N_{A}}{N_{G A R B F}} \cdot 100.0 \%
$$
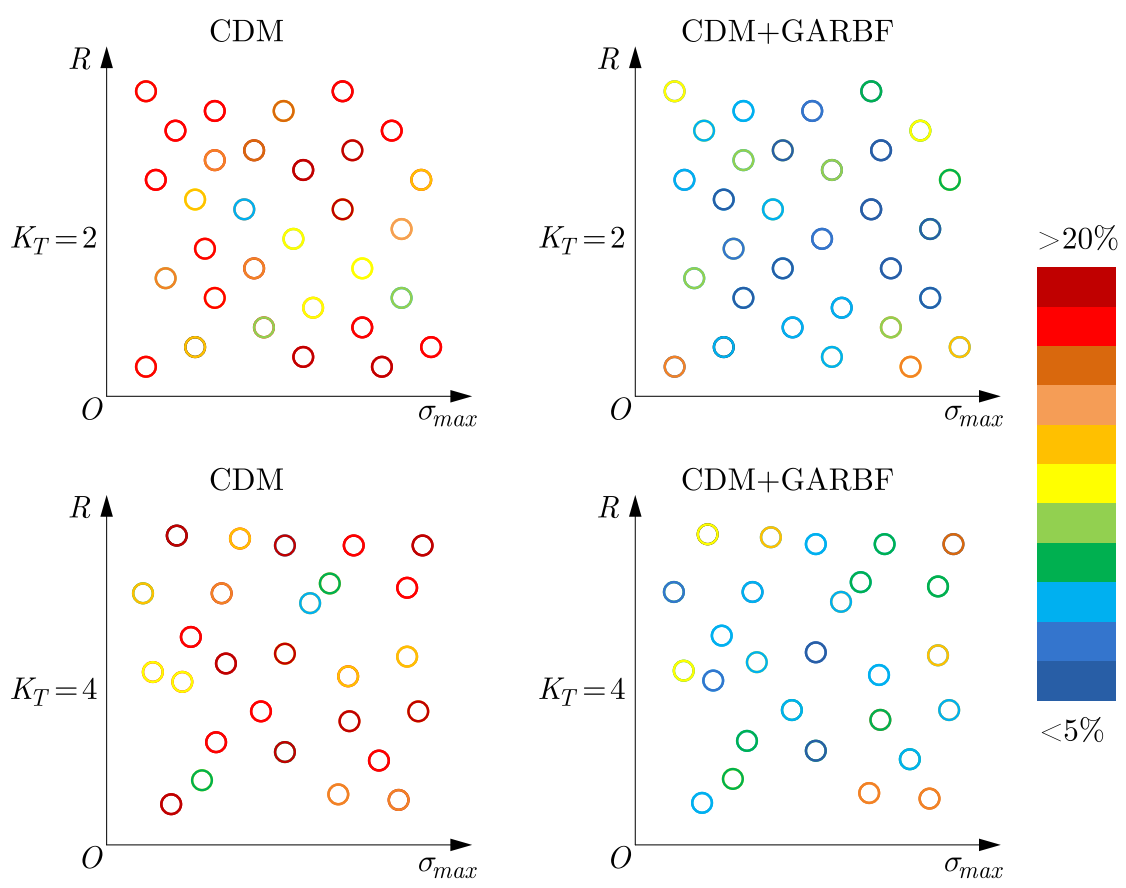

Fig. 8. The distribution of relative error calculated by CDM and CDM + GARBF

The combination method proposed in this paper is composed of a theoretical model (CDM) and an optimization model (GARBF). Actually, the selection of optimization model is not unique. In order to compare the effects of different optimization models, Table 4 respectively presents the optimization performances by traditional BPNN and GARBF models under the same verification test data. The statistic results indicate the average relative error of GARBF is $6.85 \%$, and the average relative error calculated by BPNN is $7.2 \%$. The two methods have a similar optimization effect of the average relative error. However, the GARBF model is much better than the BPNN model when comparing the variance of the relative error $\left(D_{B P}=6.0, D_{G A R B F}=2.49\right)$. Figure 9 shows the relative error optimized after BPNN and GARBF under a fixed value of $K_{T}$ and $R$. The distribution of the relative error indicates that the optimization effect of GARBF is more stable in the whole, while some calculations of BPNN seem to fluctuate significantly. The GARBF method adopts Gaussian kernel as the activation function that means the response will close to 0 when the input data is far from the cluster centers. The BPNN method uses the sigmoid function which has a large output region. Therefore, when the input data is a set of "strange 
data" that has not been trained, the output of the BPNN maybe seriously far from the true one, while the GARBF model will output a relative small value and choose to believe the results calculated by CDM theory. In a word, the optimization effect of GARBF is more reliable and stable.
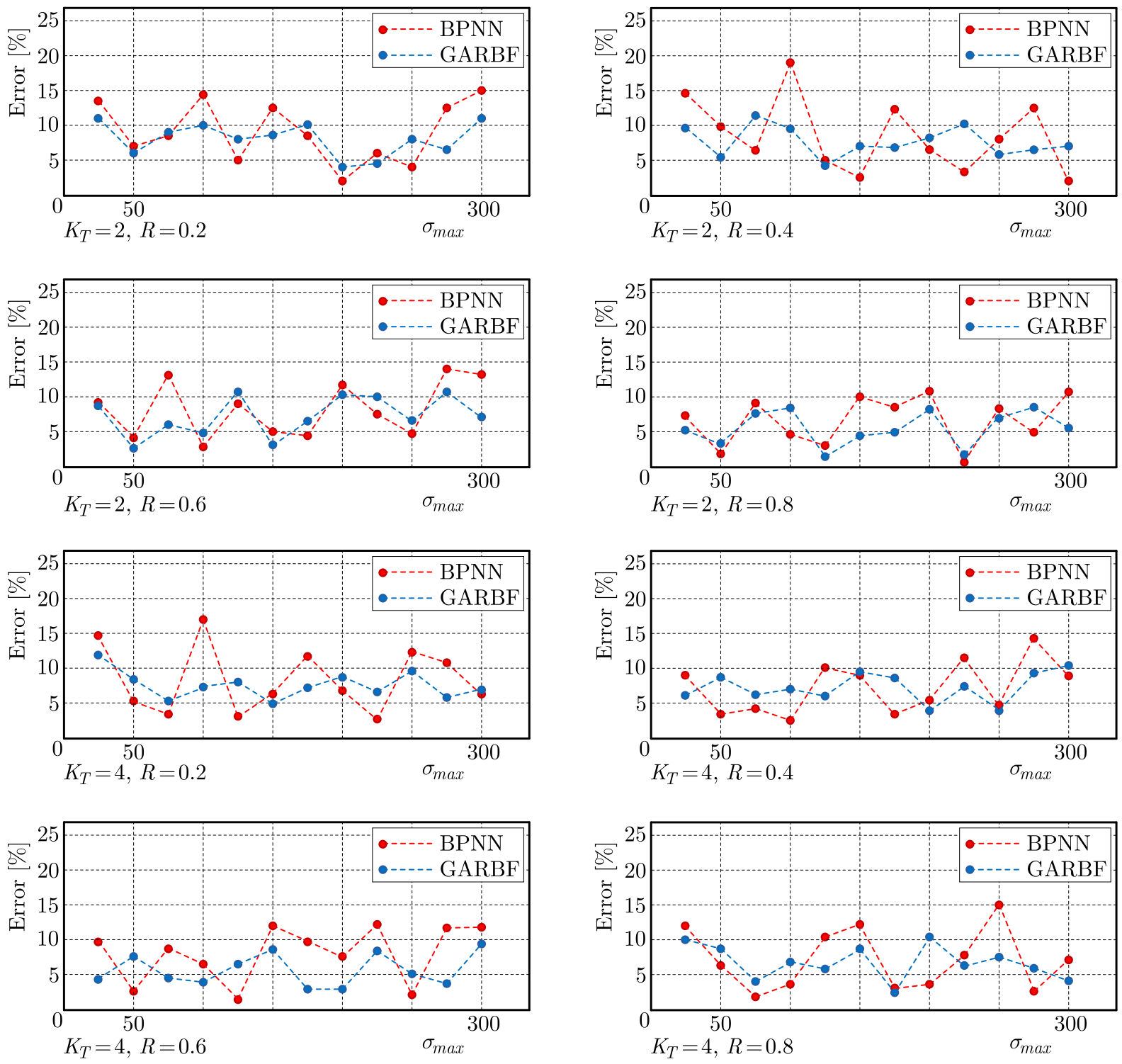

Fig. 9. The error distribution optimized by BPNN and GARBF under a fixed value of $K_{T}$ and $R$

\section{Conclusions remarks}

In this paper, a new method based on the CDM and GARBF neural network method is proposed to predict the fatigue life of LY12CZ notched plate. Some important conclusions are summarized as follows:

- The multiaxial fatigue damage evolution equation is derived and the theoretical model for fatigue life prediction of LY12CZ notched plate is established. The predicted results based on the CDM method tally with the fatigue experimental results. 
Table 4. Optimization effect comparisons between the BPNN and GARBF model

\begin{tabular}{|c|c|c|c|c|}
\hline & $\begin{array}{c}\text { Stress } \\
\text { ratio }\end{array}$ & $\begin{array}{r}\sigma_{\max } \\
{[\mathrm{MPa}]}\end{array}$ & $\begin{array}{l}\text { Relative error } \\
\text { of BPNN [\%] }\end{array}$ & $\begin{array}{l}\text { Relative error } \\
\text { of GARBF [\%] }\end{array}$ \\
\hline \multirow{10}{*}{$K_{T}=2$} & $\overline{-0.2}$ & 175 & 14.78 & 9.07 \\
\hline & -0.09 & 155 & 5.09 & 5.64 \\
\hline & 0.02 & 137 & 0.84 & 4.04 \\
\hline & 0.25 & 112 & 14.73 & 10.69 \\
\hline & 0.4 & 100 & 17.79 & 8.79 \\
\hline & 0.4 & 300 & 2.44 & 7.06 \\
\hline & 0.60 & 262 & 2.09 & 6.27 \\
\hline & 0.68 & 250 & 15.16 & 7.16 \\
\hline & 0.75 & 240 & 2.14 & 3.05 \\
\hline & 0.79 & 235 & 1.76 & 2.88 \\
\hline \multirow{10}{*}{$K_{T}=4$} & 0.02 & 137 & 10.85 & 12.03 \\
\hline & 0.25 & 112 & 16.48 & 8.48 \\
\hline & 0.4 & 100 & 3.47 & 7.66 \\
\hline & 0.55 & 90 & 2.85 & 5.64 \\
\hline & 0.60 & 87 & 6.93 & 3.95 \\
\hline & 0.60 & 262 & 2.87 & 5.41 \\
\hline & 0.68 & 250 & 3.98 & 3.83 \\
\hline & 0.75 & 240 & 15.54 & 8.54 \\
\hline & 0.83 & 230 & 1.6 & 7.55 \\
\hline & 0.91 & 220 & 2.65 & 9.27 \\
\hline \multicolumn{5}{|c|}{ Mean relative error [\%]: $e_{B P}=7.2, e_{G A R B F}=6.85$} \\
\hline \multicolumn{5}{|c|}{ Variance of relative error $\left[\%^{2}\right]: D_{B P}=6.0, D_{G A R B F}=2.49$} \\
\hline
\end{tabular}

- The RBF method is introduced to modify the relative deviation between the theoretical result and the actual life. The verification test indicates the combined method of CDM and GARBF is able to reduce the average relative error of the results of fatigue life prediction to about $7 \%$, which shows that the new method to predict the fatigue life is more reliable.

- Compared to the traditional BPNN model, the GARBF model proposed in this paper has a better optimization effect and the result is more stable. When the input is a set of "strange data" that has not been trained, the output of the BPNN may be seriously far from the true output in some local region, while the GARBF model will output a relative small value and choose to believe the results calculated by CDM theory.

\section{References}

1. Camacho-Vallejo J.-F., Mar-Ortiz J., López-Ramos F., Rodríguez R.P., 2015, A genetic algorithm for the bi-level topological design of local area networks, Plos One, 10, 6, 1-21

2. Gao J., He Q., Zhan Z., Gao X., 2016, Dynamic modeling based on fuzzy Neural Network for a billiard robot, IEEE, 13th International Conference on Networking, Sensing and Control

3. Gao J., Zhu M., Liang H., Guo X., He Q., 2015, Design of the multiple Neural Network compensator for a billiard robot, IEEE, International Conference on Networking, Sensing and Control, $17-22$

4. Goldberg D.E., Samtani M.P., 2015, Engineering optimization via genetic algorithm, Proceedings of 9th Conference Electronic Computation, ASCE, 471-482 
5. Guo H., Yin J., Zhao J., Huang Z., Pan Y., 2014, Prediction of fatigue life of packaging EMC material based on RBF-SVM, International Journal of Materials and Product Technology, 49, 1, $5-17$

6. Karolczuk A., Macha E., 2005, A review of critical plane orientations in multiaxial fatigue failure criteria of metallic materials, International Journal of Fracture, 134, 3-4, 267-304

7. Lemaitre J., Chaboche J.L., 1990, Mechanics of Solid Materials, Cambridge University Press

8. Li B., Santos J.L.T., Freitas M., 2000, A unified numerical approach for multiaxial fatigue limit evaluation, Mechanics of Structures and Machines, 28, 1, 85-103

9. LiU C.H., XUAN F.Z., 2008, A determination method of important affecting parameters on low circle fatigue life, Materials for Mechanical Engineering, 32, 12, 22-24

10. Monteiro R.L.S., Carneiro T.K.G., Fontoura J.R.A., Da Silva V.L., Moret M.A., Pereira H.B., 2016, A model for improving the learning curves of artificial neural networks, Plos One, 11, 2

11. Movaghghar A., Lvov G.I., 2012, A method of estimating wind turbine blade fatigue life and damage using continuum damage mechanics, International Journal of Damage Mechanics, 21, 6, $810-821$

12. NAGarajan R., Jonkman J.N., 2013, A neural network model to translate brain developmental events across mammalian species, Plos One, 8, 1

13. Pujol J.C.F., Pinto J.M.A., 2011, A neural network approach to fatigue life prediction, International Journal of Fatigue, 33, 3, 313-322

14. Reid D., Hussain A.J., TAWfik H., 2013, Financial time series prediction using spiking neural networks, Plos One, 9, 8, e103656-e103656

15. SchiJve J., 2001, Fatigue of Structures and Materials, Springer

16. Suresh S., 1998, Fatigue of Materials, Cambridge (UK), Cambridge University Press

17. Upadhyaya Y.S., Sridhara B.K., 2012, Fatigue life prediction: a continuum damage mechanics and fracture mechanics approach, Materials and Design, 35, 220-224

18. Wu X., 1996, Handbook of Mechanical Properties of Aircraft Structural Metals, China Aviation Industry Press, Beijing

19. Zhan Z., Hu W., Li B., Zhang Y., Meng Q., 2017a, Continuum damage mechanics combined with the extended finite element method for the total life prediction of a metallic component, International Journal of Mechanical Sciences, 124, 48-58

20. Zhan Z., Hu W., Meng Q., Guan Z., 2017b, Fatigue life and defect tolerance calculation for specimens with foreign object impact and scratch damage, Archive of Applied Mechanics, 88, 3, 373-390

21. Zhan Z., Hu W., Meng Q., Shi S., 2016, Continuum damage mechanics-based approach to the fatigue life prediction for 7050-T7451 aluminum alloy with impact pit, International Journal of Damage Mechanics, 25, 7, 943-966

22. Zhan Z., Hu W., Shen F., Meng Q., Pu J., Guan Z., 2017c, Fatigue life calculation for a specimen with an impact pit considering impact damage, residual stress relaxation and elasticplastic fatigue damage, International Journal of Fatigue, 96, 208-223

23. Zhan Z., Hu W., Zhang M., Meng Q., 2015a, A study on the effect of surface defect on the fatigue performance of metal component based on damage mechanics, Mechanics, 21, 1, 5-10

24. Zhan Z., Hu W., Zhang M., Meng Q., 2015b, Revised damage evolution equation for high cycle fatigue life prediction of aluminum alloy LC4 under uniaxial loading, Applied Mathematics and Mechanics, 36, 9, 1185-1196

25. Zhan Z., Hu W., Zhang M., Meng Q., 2015c, The fatigue life prediction for structure with surface scratch considering cutting residual stress, initial plasticity damage and fatigue damage, International Journal of Fatigue, 74, 173-182 
26. Zhan Z., Hu W., Zhang M., Zhu Y., Meng Q., 2013, Experimental method for and theoretical research on defect tolerance of fixed plate based on damage mechanics, Chinese Journal of Aeronautics, 26, 5, 1195-1201

27. Zhan Z., Meng Q., Hu W., Sun Y., Shen F., Zhang Y., 2017d, Continuum damage mechanics based approach to study the effects of the scarf angle, surface friction and clamping force over the fatigue life of scarf bolted joints, International Journal of Fatigue, 102, 59-78

Manuscript received January 14, 2018; accepted for print April 10, 2018 